\title{
INVESTIGACIONES
}

\section{Aproximación transdisciplinar a la Música Popular Urbana en Enseñanza Secundaria*}

\author{
Transdisciplinary approach to Urban Popular Music in Secondary Education
}

\author{
Carlos Lage-Gómez ${ }^{a}$ \\ ${ }^{a}$ Facultad de Educación, Universidad Complutense de Madrid. \\ calage@ucm.es
}

\section{RESUMEN}

La integración curricular representa una prioridad en el ámbito de la Educación Secundaria, con implicaciones profundas en los procesos de enseñanza y aprendizaje. Sin embargo, su aplicación en dicha etapa presenta complejidades profundadas debido a la estructura de los currículos educativos. Con el objetivo de profundizar en una comprensión transdisciplinar de la educación artística en Enseñanza Secundaria, presentamos un proyecto desarrollado en un instituto público madrileño (España). Desde el eje temático que conforman la música popular urbana, el género y la adolescencia, se ha elaborado un disco, una campaña publicitaria y un estudio de los gustos musicales del centro. Han participado 3 grupos de $4^{\circ}$ de Educación Secundaria Obligatoria (15-16 años), 3 profesores y 6 profesionales externos. Los resultados evidencian la integración transdisciplinar de diversas áreas artísticas y de ciencias sociales como herramienta útil para la Educación Secundaria, en un proceso participativo de enseñanza y aprendizaje en el que el alumnado ha construido experiencias artísticas significativas.

Palabras claves: transdisciplinariedad, música popular urbana, adolescencia, estereotipos de género, creatividad.

\begin{abstract}
Curriculum integration represents a priority in secondary education, with profound implications for teaching and learning processes. However, its application presents deep complexities due to the structuring of education systems. In order to deepen a transdisciplinar understanding of Arts Education in Secondary schools, we present a project developed in a Madrid Secondary School (Spain). From the thematic axis of popular music, gender and adolescence, an album, an advertising campaign and a musical taste map have been elaborated. All this in 3 groups of 4th year of ESO with 3 teachers and external collaboration. The results show the transdisciplinar integration of diverse art and social sciences areas as a useful tool for Secondary Schools, in a participatory process of teaching and learning, and the construction of meaningful artistic experiences by the students.
\end{abstract}

Key words: Transdisciplinarity, Popular music, Adolescence, Gender stereotypes, creativity.

\footnotetext{
* Agradecimientos. Nos gustaría expresar el agradecimiento a los participantes en el proyecto: el alumnado; los profesores Íñigo Martínez y Francisco Esquinas; el Equipo Directivo del IES Villa de Vallecas; los profesionales externos Carlos Jorge, María Rosalía, Elena Humanes y Jana Pachón, junto con el Estudio de Grabación GG Producciones.
} 


\section{INTRODUCCIÓN}

La escuela en su conjunto aborda una encrucijada que de respuesta a las múltiples y constantes transformaciones que se están produciendo en las sociedades de la información (Fernández Enguita, 2016). En este sentido, diversos sectores del ámbito universitario (Bascia \& Hargreaves, 2013), profesionales educativos (Lage-Gómez \& CremadesAndreu, 2019) o de la administración en diversos contextos internacionales (Finnish National Board of Education, 2016), promueven el replanteamiento de todos los aspectos que determinan los procesos de enseñanza y aprendizaje. En este sentido, se sugiere abordar la profundización en la transdisciplinariedad desde el ámbito artístico en la Educación Secundaria, y sus implicaciones en el replanteamiento del rol del alumnado en los procesos de enseñanza y aprendizaje creativos en el micro contexto (Bresler, 2006). Todo esto, a través de la intersección del eje transversal formado por la participación, tanto desde la perspectiva investigadora como educativa (Heron, 1996), junto con la integración artística (Cslovjecsek \& Zulauf, 2018) y las creatividades en el ámbito educativo (Glaveanu, 2018).

\subsection{LA TRANSDISCIPLINARIEDAD EN LA EDUCACIÓN ARTÍSTICA}

Desde su origen como una reacción al postpositivismo (ej. Piaget, 1973; Jantsch, 1972), la transdisciplinariedad se presenta como una aproximación a la integración del conocimiento. Pretende difuminar la atomización disciplinar para abordar la comprensión integral del conocimiento (e.j. Mittelstrass, 2011; García Díaz, 2017). En este sentido, y aunque sin una definición unívoca, la transdisciplinariedad plantearía afrontar la complejidad del conocimiento desde una perspectiva holistica e integradora como principio filosófico y paradigmático (Nicolescu, 2012). Según Hirsch Hadorn et al. (2008), la investigación transdisciplinar se centraría en: (1) abordar problemas de la vida cotidiana; (2) trasciende a paradigmas disciplinares a través de una perspectiva integradora; (3) tiene un caracter participativo, con una variedad de actores implicados de diversos ámbitos y sectores; y (4) pretende la unidad de conocimiento más allá de las disciplinas.

La transdisciplinariedad en el ámbito educativo se caracterizaría por una serie de aspectos fundamentales: (1) integración curricular en torno a diversas áreas; dicha perspectiva también se ha conceptualizado como mutidisciplinariedad, intradisciplinariedad o interdisciplinariedad (Drake \& Burns, 2004). La transcisciplinariedad profundiza en una perspectiva integradora (Marshall, 2014), que pretende difuminar las fronteras entre las áreas en una integración real de los procesos de enseñanza y aprendizaje. Todo esto desde una perspectiva metadiscipinaria y multidimensional (Morin, 1999), abriendo la puerta a una epistemología diferenciada a través de nuevos métodos y prácticas (Klein et al., 2001). Según Marshall (2014, p. 106): “en el ámbito de la educación artística, la integración favorece la adquisición de habilidades procedimentales y conceptuales, así como la metacognición". (2) Holismo y unidad del conocimiento desde la teoria general de sistemas como principio epistemológico; un sistema quedaría definido como un conjunto de elementos en interacción y relacionados entre si (Bunge, 1980). Dicha concepción se correspondería con la perspectiva de Morin (1999) en el ámbito educativo, para quien la integración y la complejización del conocimiento se convierte en una cuestión esencial: "un conocimiento fragmentado según las disciplinas impide a menudo operar el vínculo entre las partes y las totalidades y debe dar paso a un modo de conocimiento capaz de aprehender 
los objetos en sus contextos, sus complejidades, sus conjuntos" (Morin, 1999, p. 2). (3) Conectar el aprendizaje con el mundo real; la transcisciplinariedad implicaría abordar problemas reales, complejos y socialmente relevantes (Burger \& Kamber, 2003), y que permita alcanzar soluciones sostenibles (Soublis Smyth, 2017). (4) Diversos participantes implicados de distintos ámbitos y sectores; además, el rol del alumnado es activo (Park \& Son, 2010), colaborando en la toma de decisiones en los procesos de enseñanza y aprendizaje, y asumiendo una variedad de roles (Adams, Gibson \& Muller, 2008).

Desde el punto de vista de la integración de las artes, Dewey (1934) propone una aproximación experiencial, holística y humanizadora, relacionada con la perspectiva integradora de Sorieau (1998): “el arte es lo que existe de común entre una sinfonía y una catedral, una estatua y un ánfora; es lo que hace equiparables entre sí la pintura y la poesía, la arquitectura o la danza" (Sorieau, 1998, p. 3). Entre las experiencias centradas en la integración a través del arte, cabría mencionar el European Language Portfolio (http:// www.kielisalkku.edu.fi/fi), que aborda el aprendizaje conjunto entre la música y las lenguas (Marjanen \& Cslovjecsek, 2013), o una aproximación posterior que aborda la integración curricurar entre la música y las matemáticas (Viladot et al., 2018). La metodología STEAM (Perignat \& Katz-Buonincontro, 2019), se propone la integración entre las artes y las áreas científico-tecnológicas (matemáticas, ciencias, tecnología e ingeniería). También, el proyecto LOVA (https://proyectolova.es/) en España, aborda la integración curricular y artística a través de la creación de óperas escolares. Asimismo, existen numerosos proyectos en centros a lo largo del mundo que abordan la integración curricular a través de las artes.

\subsection{PARTICIPACIÓN EN EDUCACIÓN MUSICAL Y ESPACIOS DE APRENDIZAJE ARTÍSTICOS}

Desde el origen platónico de la participación, definida como un concepto metafísico que relaciona lo sensible con la Idea, Santo Tomás de Aquino profundiza dicha conceptualización al considerar que el ser participa en todo ente según su propia naturaleza. En el ámbito educativo, la participación ha sido abordada en la literatura como (1) un motor para llevar la democracia al corazón mismo de la escuela (Dewey, 1995), o (2) a través de su vínculo con la motivación y satisfacción en el aula (Withall, 1989). Freire (2003) considera la participación como tener voz y determinados grados de decisión. Desde dicha perspectiva, se podría considerar que existen diversas dimensiones en la participación en el aula que oscilan desde su consideración como una estrategia docente, la que favorece el aprendizaje del alumnado, hasta su concepción como un fin educativo en sí mismo. Dos factores clave se podrían identificar para definir la participación en el aula desde dicha perspectiva: (1) la toma de decisiones por parte del alumnado y (2) los diferentes grados en los que esta se podría producir. Así pues, la participación del alumnado podría ser analizada a través de todas las fases de los procesos de enseñanza y aprendizaje que se desarrollan en el aula. La participación como la capacidad de decidir por parte del alumnado en: (1) el diseño curricular, (2) los procesos de aprendizaje y (3) los productos resultantes. El rol del alumnado en los procesos de enseñanza y aprendizaje representa un tema trascendente abordado en la literatura, desde la tradicional dicotomía entre pedagogías centradas en el alumnado o en el profesorado. Wegerif (2019) se refiere a la educación dialógica como centrada en el diálogo, ni en el estudiante ni en el profesorado. Así, las pedagogías centradas en el alumnado se han basado tradicionalmente en la experiencia e intereses del alumnado a través de procesos participativos y colectivos o cooperativos tendentes 
fomentar la democracia en el aula y centrados en el diálogo y la empatía con el objetivo de motivar al alumnado. En este sentido, se abriría la comprensión de la participación desde otra vertiente, que implicaría una adecuación de lo individual a lo social como una forma de compartir en lo común.

\subsection{CREATIVIDADES EN EDUCACIÓN}

Durante la primera década del siglo XXI se ha puesto de manifiesto una reconceptualización de la creatividad en el ámbito educativo, no solo como un fenómeno cognitivo complejo que tiene lugar en el indivíduo (Feldman, Csikszentmihalyi \& Gardner, 1994), situado en un contexto social y cultural (Csikszentmihalyi, 1999), sino como una forma de pensamiento en posibilidades (Burnard et al., 2015), que emerge como una práctica social a través de diversas formas (Burnard, 2012; Glaveanu, 2018; Sternberg, 2005). Desde esta perspectiva, se han enfatizado: (1) la colaboración desde su perspectiva social y distribuida (Miell \& Littleton, 2004; Sawyer \& DeZutter, 2009); (2) la inclusión en procesos de aprendizaje transformadores (Lapidaki et al., 2012); (3) la interculturalidad, como construcción cultural (Saether, Mbye \& Shayesteh, 2012); (4) la empatía en las interacciones grupales (Seddon, 2012; Cross, Laurence \& Rabinowitch, 2012); (4) la participación como elemento multidimensional en los procesos creativos (Lage-Gómez \& Cremades-Andreu, 2020).

En el presente artículo se presenta el informe de un estudio de Investigación Acción desarrollado en un centro de Enseñanza Secundria con alumnado de $4^{\circ}$ de Educación Secundaria Obligatoria (15-16 años) de la ciudad de Madrid (España). Se ha pretendido profundizar sobre una comprensión transdisciplinar de la Música Popular Urbana, para lo que se han planteado las siguientes preguntas de investigación:

¿Cómo se produce la integración transdisciplinar en el proyecto didáctico centrado en la música popular urbana?

¿Cómo influye en el desarrollo del proyecto didáctico el papel activo y centrado en la participación del alumnado?

¿Qué elementos caracterizan el aprendizaje en el proyecto didáctico centrado en la aproximación transdisciplinar a la música popular urbana?

\section{MÉTODO}

Freire (2003) sitúa la transformación social como eje que vertebra la acción educativa desde todas las áreas, y considera que la investigación educativa emerge cómo y en una práctica social que emana de la experiencia. Dicha perspectiva social y vertebradora conectaría, a su vez, con una aproximación transdisciplinar aplicada a la educación, una forma de ir más allá de lo puramente disciplinar con una voluntd transformadora (Acaso, 2013). Así, se ha desarrollado un proyecto de Investigación Acción desde nuestro rol docente e investigador (Cochran-Smith \& Litle, 2009), convencidos de la importancia de la comprensión de la realidad educativa desde dentro (Burnard, Apelgren \& Cabaroglu, 2015), en colaboración con profesionales de diversos ámbitos.

Desde una perspectiva analítica deductiva, se ha recurrido a la Teoría de la actividad (Engeström, 1999) como marco analítico que nos ha permitido una mejor comprensión de la gran variedad de elementos relacionados en las tareas en las que se encuentran involucradas 
un grupo social. Dicha perspectiva ha sido empleada en el ámbito de la educación musical para el análisis de las características propias de las actividades de composición cooperativa (ej. Bournard y Younker, 2008), improvisación colectiva (Burnard \& Dragovic, 2014) o del fenómeno multifacético que representa la composición utilizando soportes informáticos por adolescentes (Soares, 2012). Partiendo de la tradición social-histórica rusa de Vigotsky, Engeström (1987) proporcionó un modelo para realizar el análisis de las actividades y representarlas a través de una unidad de análisis definida como sistema de actividad.

Figura 1. Teoría de la Actividad de Engeström

(Engeström, 1999) (Elaboración propia).

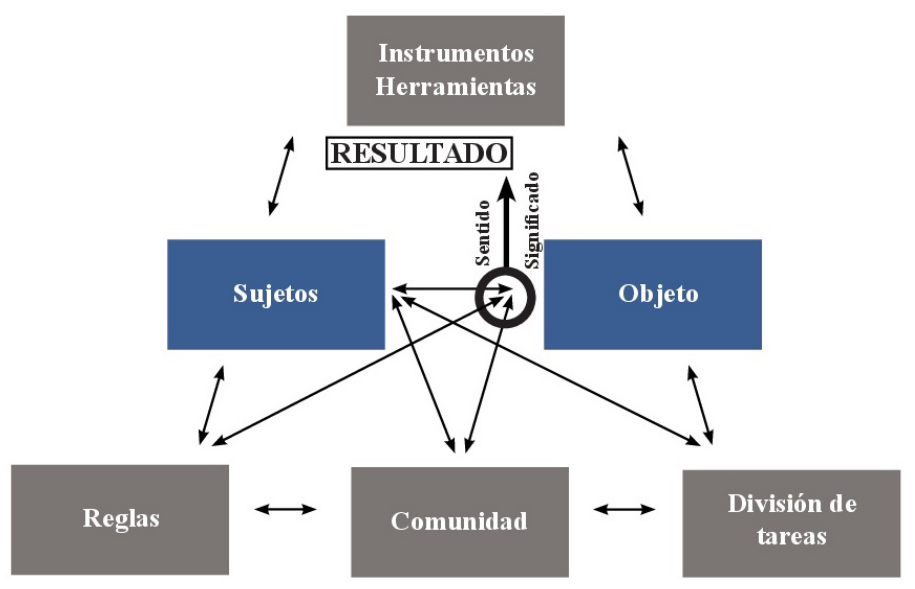

Desde nuestra perspectiva investigadora, y a través de los procesos de enseñanza (Greeno \& Engeström, 2014), nos propusimos comprender y explicar los procesos de aprendizaje del alumnado en una propuesta artística transdisciplinar. Así, y en nuestro caso, el sujeto estaría representado por el alumnado que participa en el desarrollo de un proyecto didáctico centrado en la música popular urbana, y definido como el objeto. La relación en entre el sujeto y el objeto se encuentra mediada por una serie de instrumentos o herramientas, en este caso centradas en la transdisciplinariedad, junto con una serie de reglas derivadas de la implicación, participación activa y motivación de los participantes, así como la división de trabajo, a través de los diversos roles asumidos por el alumnado, junto con el aprendizaje cooperativo. 


\section{EL PROYECTO DIDÁCTICO}

\subsection{EL CONTEXTO}

El centro, un instituto público de Educación Secundaria, se encuentra ubicado en la ciudad de Madrid (España). Un elemento característico que nos permite contextualizar y aproximarnos a las características del alumnado desde un punto de vista sociológico, representa el hecho de que se trata de uno de los 6 de los 21 distritos de la ciudad con una mayor tasa de paro y una menor renta per cápita (CAM, 2018). El centro destaca por su heterogeneidad y diversidad; hace algunos años fue catalogado como "centro público prioritario" y en la actualidad sigue siendo reconocido como de "difícil desempeño". Aproximadamente un $31 \%$ del alumnado procede de 26 países distintos, con un índice de suspensos muy elevado en el momento de la realizaciòn del estudio.

\subsection{PARTICIPANTES}

En el proyecto didáctico participaron tres grupos, uno del área de Música y dos de Educación Plástica y Visual. Un total de 74 alumnos/as, 46 chicas y 28 chicos, estuvieron involucrados en el proyecto. El área de música estaba integrada por alumnado de dos grupos; uno de ellos, considerado por el equipo docente como un muy buen grupo, tanto desde el punto de vista de los resultados académicos como actitudinal. El segundo grupo, del que el profesor de Música participante fue su tutor, fue calificado de forma genérica por el equipo docente como un grupo con un nivel académico bajo. En el proyecto didáctico participaron además otros dos grupos del área de Educación Plástica y Visual de $4^{\circ}$ de ESO, definidos en las juntas de evaluación como grupos complejos y con resultados académicos bajos. Se pone de manifiesto así, lo que se podría calificar como gran una heterogeneidad en el aula, explicitada por el propio alumnado en las clases al establecer una clara dicotomía, tanto en el plano personal como colectivo, entre lo que ellos mismos definían como "los buenos y los malos" (Diario de clase, Profesor). Esta situación, con claras implicaciones en la dinámica y ambiente del aula durante los primeros meses, se fue diluyendo a medida que avanzaba el curso y evolucionaba el proyecto.

Además, participó el grupo de cine del centro. Se trataba de un grupo de seis alumnos/ as de $3^{\circ}$ de Educación Secundaria Obligatoria, que bajo la supervisión de un profesor del centro realizaban cortometrajes cinematográficos. Con el objetivo de involucrar a un abanico amplio de participantes en un proyecto de centro, contamos con su colaboración para la grabación de un audiovisual para la campaña publicitaria del disco, en forma de making-of.

El proyecto fue implementado por tres profesores del centro de los Departamentos de Música, Dibujo y Educación Física, con el apoyo del equipo directivo del centro, así como del Departamento de Orientación Educativa. Con el objetivo de establecer un puente entre el ámbito educativo y la actividad profesional que se desarrolla fuera del mismo, nos pareció muy oportuno solicitar la colaboración de una serie de instituciones y profesionales expertos en diversas áreas relacionadas con el proyecto que pudieran aportar su perspectiva al alumnado, así como actuar como agentes motivadores para el mismo. En este sentido, se contó con la colaboración de dos Agentes de Igualdad del Ayuntamiento de Madrid (España), que han colaborado en el diseño y realización de la investigación sobre los estereotipos de género en la música popular, concretadose en una primera fase 
de entrevistas colectivas con el alumnado. En relación con el ámbito de la comunicación y difusión, se contó con la colaboración del director creativo de una empresa de reconocido prestigio que ha impartido una conferencia al alumnado sobre la temática de la publicidad, así como supervisado con ellos/as la realización del logo, etc. Por último, se colaboró con un estudio de grabación profesional para la grabación del disco.

\subsection{UPSTAIRS: ALWAYS GOING UP. EL PROYECTO}

El proyecto didáctico transdisciplinar estuvo guiado por tres aspectos vertebrados entre sí: la música popular urbana, los estereotipos de género y la adolescencia. Desde el punto de vista general nos planteamos desde los procesos de aprendizaje: (1) potenciar el pensamiento creativo del alumnado a través de la creación musical y artística, así como de la investigación; (2) concienciar al alumnado sobre la influencia de los estereotipos de género en la música que escuchan habitualmente; (3) incrementar la motivación del alumnado ante el aprendizaje, desde una perspectiva holística e inclusiva, favoreciendo la consecución de experiencias significativas; (4) dotar al alumnado de un papel activo en su propio aprendizaje, involucrándolo en la toma de decisiones de cada fase del proyecto; (5) familiarizar y acercar a este a la creación artística, desde los planos interdisciplinares, globales e integradores.

El proyecto didáctico se desarrolló a lo largo de todo el curso 2014/2015 a través de diversas fases. La fase de elaboración del disco, tanto los arreglos como la composición, se desarrolló durante la primera y la segunda evaluación del curso, a lo largo de 52 sesiones. La fase de investigación sobre estereotipos de género y de elaboración del mapa musical del centro se ha producido durante la tercera evaluación del curso, a lo largo de 32 sesiones. La campaña publicitaria se produjo durante la tercera evaluación, a lo largo de 26 sesiones.

\subsubsection{La elaboración del disco}

Se elaboró un disco con seis temas arreglados y uno compuestos por el alumnado. El arreglo de los temas se produjo de forma colectiva en el aula de música a través de distintas fases. Todos los temas fueron elegidos por el alumnado a través de las fases de propuesta, votación y acuerdo respectivamente. Así, tras las propuestas iniciales por parte del alumnado, se realizaban votaciones sucesivas hasta alcanzar acuerdos que contaran con al menos el $80 \%$ del alumnado. Esta fue la norma que acordamos en el aula al comienzo del proyecto. Los temas fueron arreglados e interpretados con los instrumentos disponibles en el aula (guitarras eléctricas, bajo eléctrico, batería, teclados, xilófonos y metalófonos) y vocalmente. El alumnado eligió el rol en función de sus propias preferencias, y procurando que fueran cambiando en los distintos temas musicales. Los procesos de aprendizaje se produjeron a través de la guía y propuestas iniciales por parte del docente, tomando como referencia los temas originales. Posteriormente, se acordaban en el aula todos los aspectos referidos a la estructura, armonía e instrumentación de los temas. En este sentido, los resultados no han sido una prioridad en el proyecto, sino más bien su carácter inclusivo.

En la segunda fase del proyecto se establecieron grupos de trabajo del alumnado de música para el diseño y desarrollo de la investigación sobre los estereotipos de género en las músicas populares a través del mapa de gustos musicales del centro y para la creación del tema para el disco. Finalmente, los temas fueron grabados con la colaboración de un estudio profesional de música. 


\subsubsection{Campaña publicitaria: El logotipo, las camisetas, los carteles y la carátula del CD}

La campaña publicitaria se desarrolló a través de distintas fases. En primer lugar y de forma colectiva en ambas clases se produjo la realización de las predicciones a través del estudio de necesidades y briefing. Para esto, se estableció una toma de contacto entre ambos grupos y con el grupo musical. En segundo lugar, se establecieron los objetivos a través de la concreción publicitaria mediante los diseños del logotipo, carteles, camisetas, página web y vídeo promocional o making-of del disco. A continuación, se establecieron grupos de trabajo cooperativo para la realización del diseño del logotipo en primer lugar. Así, se realizaron una serie de bocetos que fueron posteriormente digitalizados. En dicho proceso, se fotografiaron y grabaron algunos de los ensayos del grupo de música que posteriormente se tomaron como modelo para la elaboración tanto del logotipo como de los carteles, camisetas, etc. En dicho proceso contamos con la colaboración de un experto y profesional del ámbito audiovisual.

\subsubsection{La elaboración del mapa musical del centro}

Con el objetivo de abordar la temática de la música en la adolescencia, nos planteamos proponer al alumnado que investigara sobre la música y la adolescencia desde la perspectiva de los estereotipos de género. Así, nos pareció oportuno que el alumnado concretara el tema a investigar, el cual, tras un proceso de debate y reflexión con la supervisión del profesor de música, se concreto en la elaboración de un mapa de gustos musicales del centro. El alumnado elaboró un cuestionario que posteriormente fue supervisado por un investigador en ciencias sociales externo al centro. En una segunda fase, el alumnado implementó el cuestionario por todos los grupos de segundo, tercero y cuarto de la ESO, así pues, se obtuvo una muestra de más de 452 alumnos/as n=452 del total. La tercera fase consistió en el volcado y categorización de este.

\subsection{INSTRUMENTOS}

En el desarrollo del estudio se emplearon diferentes técnicas e instrumentos de recogida de datos: (a) observación participante plasmada en los diarios de clase; (b) observación no participante reflejada en informes de observación; (c) cuestionario; (d) entrevistas individuales y colectivas. Desde la concepción de profesores investigadores se realizó la observación participante, reflejando tras cada una de las sesiones, la evolución, actitud, progreso y pensamientos de cada momento en un diario de clase de los profesores participantes. La observación no participante fue desarrollada por una docente del centro. Además, se elaboró un cuestionario de tipología abierta y cerrada, según la escala de Likert (Mucho, bastante, algo, poco, nada), adaptado de Lage-Gómez (2016), para obtener la perspectiva del alumnado sobre los procesos de aprendizaje. También, se ha recabado la perspectiva de los profesionales participantes a través de entrevistas semi-estructuradas.

\subsection{PROCEDIMIENTO}

Desde el marco deductivo de la Teoría de la Actividad, descrito con anterioridad, el análisis cualitativo de los procesos de aprendizaje a lo largo de las diversas sesiones plasmados en 
los diarios de clase y los informes de observación realizados por los docentes, han sido triangulados con las entrevistas y el análisis cuantitativo del cuestionario, a través de un proceso iterativo de lectura y relectura de los datos (Rice \& Ezzy, 1999).

\subsection{ASPECTOS ÉTICOS}

El proyecto se llevó a cabo en colaboración con el alumnado y demás participantes. En primer lugar, se expuso al alumnado la posibilidad de la realización del proyecto. Tras su respuesta satisfactoria se solicitaron los permisos pertinentes de la dirección del centro y de la jefatura de estudios. Asimismo, se informó y solicitó el consentimiento informado a las familias para la grabación en vídeo y la toma de imágenes.

\section{RESULTADOS}

Los resultados que se presentan a continuación evidencian la triangulación de los datos obtenidos en torno a las preguntas de investigación.

\subsection{LA TRANSDISCIPLINARIEDAD ARTÍSTICA}

A lo largo del proyecto se ha abordado el universo de la música popular urbana a través de la conexión explícita con una serie de aspectos que la conforman como han sido:

(1) La interpretación musical

Figura 2. Videocaptura del audiovisual elaborado

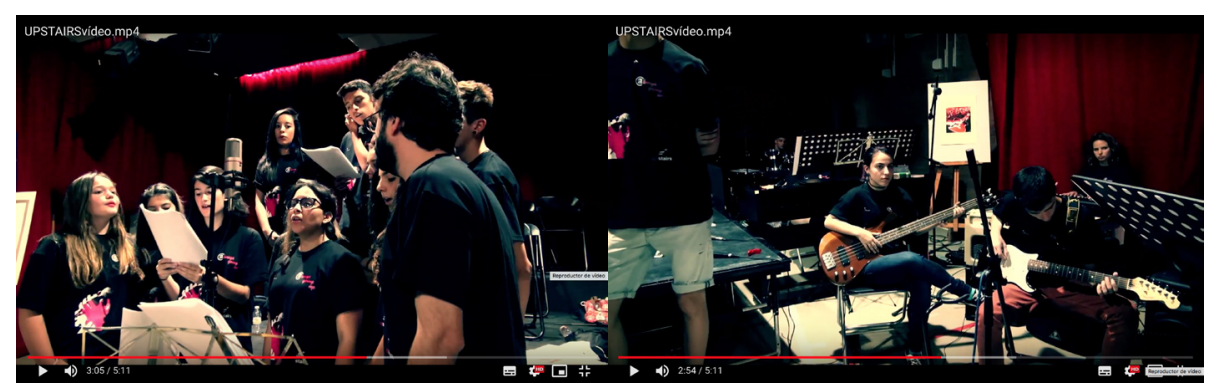


Estudios Pedagógicos XLVI N 2 : 159-176, 2020

APROXIMACIÓN TRANSDISCIPLINAR A LA MÚSICA POPULAR URBANA EN ENSEÑANZA SECUNDARIA

(2) La creación musical

Figura 3. Fotografía tomada por un alumno para la sesión de Briefing que refleja el proceso creativo en el aula de música.

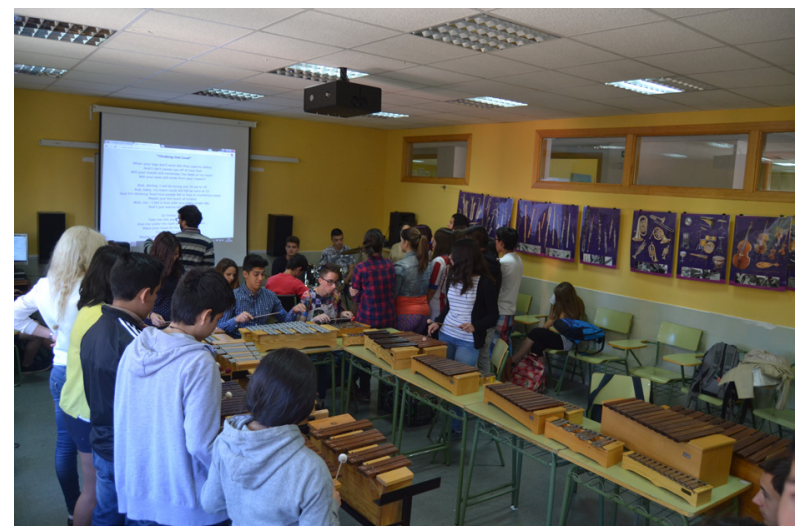

(3) Elaboración de un logo y campaña publicitaria

Figura 4. Carátula del CD y página web elaborada por el alumnado

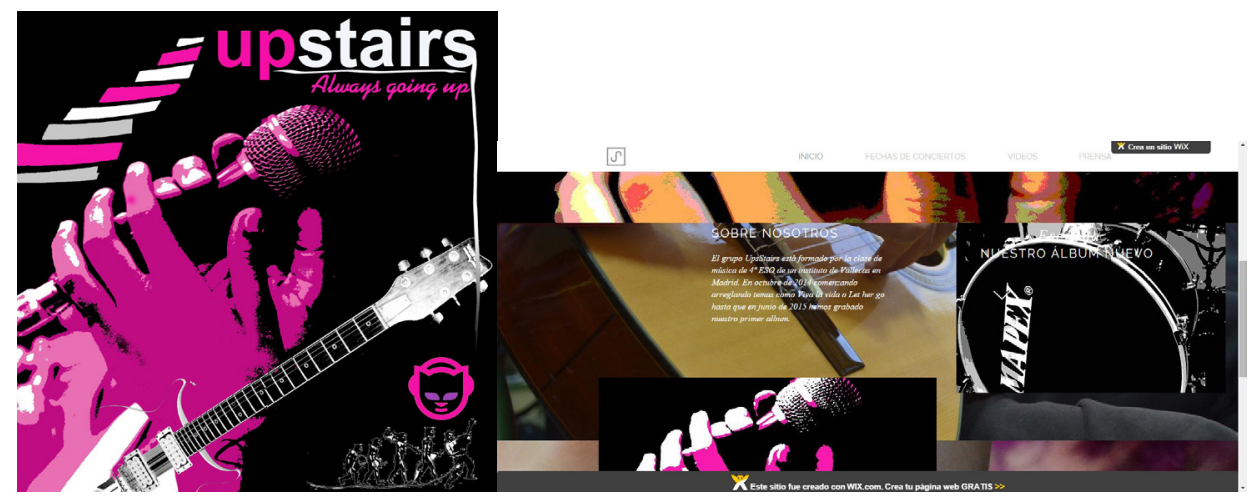


(4) Grabación del disco y un vídeo resumen del grupo

Figura 5. Fotografía tomada por un alumno durante del proceso de la grabación del disco y audiovisual del grupo.

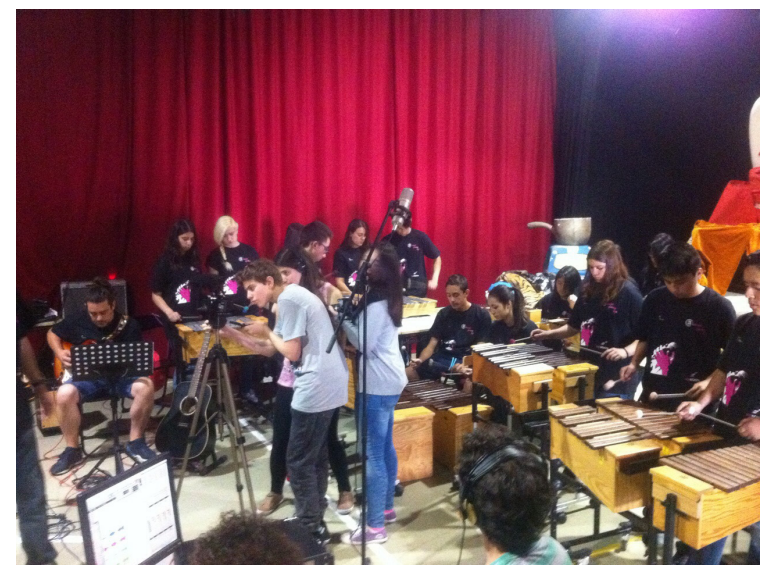

(5) Elaboración de un mapa de gustos musicales del centro

Figura 6. Muestra de los resultados del mapa de gustos musicales elaborados por el alumnado.

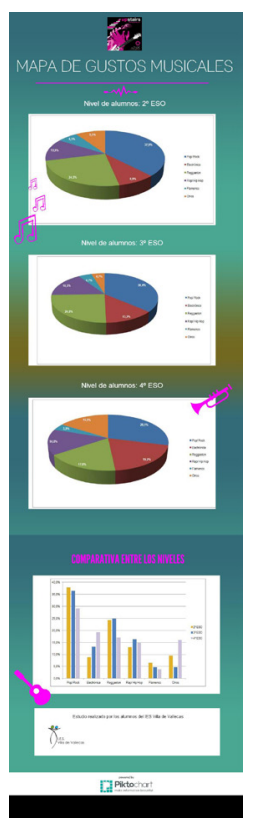


(6) La igualdad de género en la música popular urbana.

Figura 7. Coloquio con un experto colaborador en el proyecto.

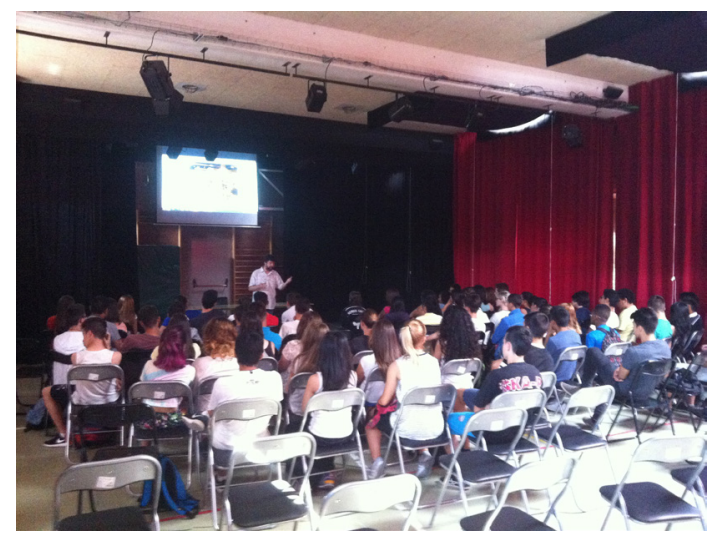

La triangulación de los datos refleja cómo el alumnado ha co-construido los procesos de aprendizaje desde su propia experiencia y a través de: (1) La experimentación; (2) Las sugerencias explícitas o implícitas por parte de los docentes; (3) El intercambio de ideas en el grupo a nivel colectivo; (4) Las contribuciones específicas del almnado.

Se ha producido la integración artística desde un punto de vista curricular a través de las asignaturas de Música y Educación Plástica y Visual para abordar el fenómeno de la música popular urbana. El alumnado ha asumido una serie de roles: intérpretes, creadores, diseñadores gráficos, artistas audiovisuales o investigadores. Todo esto nos ha permitido difuminar las fronteras de las áreas en una perspectiva multidimensional e integradora hacia una perspectiva holística de la aproximación de la música popular urbana en el ámbito de la Educación Secundaria. Es decir, hemos abordado la complejización del tema a través de la comprensión de una serie de aspectos conectados con la música popular urbana. Para todo ello, han participado de forma activa junto con el alumnado y profesorado, una serie de profesionales que han aportado una perspectiva diferenciada en la comprensión y complejización del tema, y acercarlo así al mundo real. Asimismo, la igualdad de género, que emerge como un problema real y socialmente relevante cuya presencia en la música popular urbana se hace visible ha sido tratado a lo largo del proyecto. En relación con la sensibilización del alumnado sobre los estereotipos de género, se ha producido un aprendizaje en el alumnado participante sobre los roles diferenciados entre los hombres y las mujeres, y cómo ellos se expresan y se retroalimentan en las letras de las canciones que escuchan a diario. En este sentido, las entrevistas colectivas realizadas han posibilitado tratar los estereotipos de género en la música popular, y la percepción del propio alumnado; el grado de importancia que le otorgan y cómo puede influir en sus percepciones y conductas, tanto implícitas como explícitas.

Se ha abordado la importancia de la música como parte del proceso de socialización y de construcción de la identidad individual y colectiva, y por tanto cómo los mensajes que se transmiten inciden y condicionan su forma de entender el mundo desde patrones sexistas. Es 
decir, que los estereotipos moldean la personalidad y se atribuyen unos roles diferenciados por el sexo, dándole al masculino el rol principal y al femenino uno subordinado.

Un valor añadido del proyecto ha sido la interrelación entre el proceso de reflexión descrito, las actividades desarrolladas y su repercusión en la sensibilización del alumnado sobre los estereotipos de género, que se observa en dos planos: (1) se pone de manifiesto un mayor interés, capacidades y herramientas para "decodificar" los mensajes que se transmiten en las letras de las canciones e imágenes de los videos musicales; (2) se incide en el desarrollo de comportamientos y actitudes no sexistas del alumnado en las actividades del proyecto. Han tenido la misma valía, responsabilidades, obligaciones y derechos sin condicionamientos de género, rompiendo con la tendencia a que los chicos y las chicas participaran de forma más activa en unas u otras actividades, según roles y estereotipos de género generalmente asumidos.

Además, y en relación con el aprendizaje y la perspectiva aportada por el alumnado para corroborar lo apuntado en el cuestionario: el 44,8\% considera que ha aprendido mucho"; el 41,4\%, bastante; y el 13,8\%, algo. En relación con el grado de cumplimiento de las expectativas iniciales, lo que en cierta medida se asocia con la autopercepción del éxito del proyecto. El 48,3 \% considera que las expectativas iniciales se han cubierto "mucho"; el 37,9, bastante; y el 13,8, algo.

\subsection{LA PARTICIPACIÓN DEL ALUMNADO EN EL PROYECTO}

Se ha tratado de propiciar la participación activa del alumnado en la decisión de todos los aspectos que han formado parte del proyecto, desarrollando prácticas de democracia participativa. Se ha puesto de manifiesto la consideración del desarrollo del proyecto como paulatino, aunque con altibajos, y constante; aun así, su dirección podría ser definida como ascendente y vinculada con los resultados parciales atisbados por el alumnado. Es cierto, no obstante, que los docentes y el propio alumnado han detectado algunas dificultades en el trabajo cooperativo, en relación con la participación de todos los integrantes del grupo o la "sensación de cierto caos" (Diario de Clase, Profesor), en algunos momentos. Este aspecto, en paralelo con la consideración por parte del 100\% del alumnado y profesorado del buen ambiente del aula, señalado a su vez, como uno de los elementos determinantes del proyecto. Los motivos se podrían encontrar, entre otros, en la satisfacción con las actividades propuestas. Han valorado positivamente el buen ambiente, la importancia que ha tenido para ellos expresar, de un modo libre y en la práctica, muchos de los parámetros musicales que conocían o estaban aprendiendo sobre la marcha. Así, el carácter colectivo del aprendizaje ha sido uno de los aspectos señalados por el propio alumnado como fundamental. El $80 \%$ se ha referido a aspectos de la participación en el cuestionario; en este sentido, un alumno señalaba en el cuestionario: "he aprendido a tener más empatía con la gente, también a trabajar en grupo, porque a mí no me gustaba trabajar en grupo, pero este año me he dado cuenta de que es incluso mejor" (Cuestionario, alumno/a 1), mientras que otro alumno/a se refería a la importancia de "respetar y contrastar las diversas opiniones" (Cuestionario, alumno/a 2), o "escuchar a los demás a escuchar opiniones distintas a las mías" (Cuestionario, alumno/a 3).

Así, el aprendizaje a partir del caos ha sido característico en determinadas fases del proceso, considerándolo como propio, entre otros, del aprendizaje informal. No obstante, este lo podríamos definir como dirigido hacia los procesos de aprendizaje, desde un punto de vista general. Los diferentes grupos han organizado de forma autónoma el tiempo y el 
espacio disponible en algunas fases del proyecto Así, desde la intuición y la negociación constante, tanto explícita como implícita, el alumnado ha dotado de significado su propio aprendizaje. Así, un alumno señalaba en el cuestionario cómo "he aprendido a ser mejor compañera, y a aprender a escuchar a todos independientemente del comentario que sea, ya que todos aportan algo y hacen que sea mejor" (Cuestionario, alumno/a 4). La perspectiva explícita se ha conformado a través de procesos de comunicación verbal, mientras que la implícita ha tenido lugar mediante una forma de comunicación no verbal y específicamente musical a través de la interpretación, "con un nivel elevado de implicación y concentración" (Informe de observación, observadora no participante).

Hemos corroborado cómo el proceso creativo se ha producido de forma participativa, como una forma de compartir en lo común, es decir, el resultado no ha sido la suma individual de las partes, sino que, como creación colectiva, esta ha emergido del grupo desde su consideración de unidad creativa y ha ido más allá de la suma individual de sus partes, hasta alcanzar, un sentido de pertenencia por parte de todo el alumnado a su grupo: UpStairs. Aunque el rol del alumnado variaba en función de múltiples factores como habilidades específicas o motivación, han sido conscientes de cómo cada una de ellas era fundamental para el resultado final. Así, la identidad individual se ha diluido durante el proceso en una identidad colectiva a través de su propia música. Se ha puesto de manifiesto la importancia del papel activo del alumnado en su propio aprendizaje, al que se ha dotado de significado. En este sentido, han valorado especialmente la creación y la interpretación de sus propias obras, como elemento de identidad tanto individual como colectiva. El alumnado ha asumido su cometido en el grupo, más allá de su mayor o menor participación. Se ha evidenciado la influencia de los resultados, a nivel parcial y en el producto final, como un agente motivador y elemento que ha favorecido la identificación de los grupos en torno a sus creaciones, es decir, las creaciones de los grupos han beneficiado el desarrollo de una unidad colectiva, ya que el alumnado las ha incorporado como propias. En este sentido, el alumnado muestra un elevado grado de satisfacción con los resultados obtenidos: el 65,5\% ha respondido mucho; el 31\%, bastante; y el 3,4, algo. En definitiva, los alumnos/as han construido experiencias significativas a partir de sus vivencias en el aula desde el rol que han asumido como creadores e intérpretes.

\subsection{MOTIVACIÓN Y EMOCIONES POSITIVAS EN EL AULA}

Podemos afirmar que el alumnado permaneció durante todo el proyecto con un elevado nivel de motivación, reflejado en sus actitudes individuales y colectivas, en sus declaraciones, y el entusiasmo con el que emprendían todas las actividades propuestas, así como en la participación atenta y creativa que se reflejaba en las sesiones en las que se desarrolló este trabajo. Por otra parte, su empeño cuando se proponía repetir algo para mejorarlo: He aprendido a que si le dedicas tiempo y te esfuerzas por hacer lo que quieres lo puedes conseguir (Alumno/a, cuestionario). En este sentido, el 48,3\% ha indicado un nivel muy elevado de motivación; el 44,8\%, bastante; y el 6,9, algo; lo que sin duda ha sido un "catalizador" que se visibiliza en términos de procesos (dinámicas de trabajo colectivo) como de resultados (tanto en la autopercepción de los y las alumnas como en las valoraciones del equipo docente).

Hemos sido conscientes de cómo han emergido emociones positivas en el aula, tanto en el plano individual como social. Se ha podido corroborar la influencia de: (1) un 
grado elevado de indentificación con el proyecto, a través del compromiso con el mismo, establecido como una meta compartida por el grupo; (2) la significatividad del mismo para el alumnado; (3) el ambiente positivo generado en el aula. El alumnado se encontraba cómodo y relajado, algo que propició climas apropiados para el aprendizaje.

\section{DISCUSIÓN E IMPLICACIONES EDUCATIVAS}

La implementación del proyecto nos ha permitido promover un proceso transformador en el aula, tanto desde la perspectiva del aprendizaje como de la enseñanza, y a través de una perspectiva transdisciplinar de la música popular urbana. Así, y en línea con otros proyectos desarrollados a través de presupuestos artísticos transversales desde la perspectiva de profesionales investigadores (e.g. Lage-Gómez \& Cremades-Andreu, 2018), hemos comprobado el éxito del mismo, debido a su carácter auténtico, con la participación de un amplio abanico de agentes implicados, inclusivo y reflexivo, en el que distintos lenguajes se han fundido en una única manifestación artística. Precisamente, su carácter transdisciplinar, tal y como sugiere Marshall (2014), se ha convertido en un elemento que ha promovido (1) un aprendizaje integrado de todos los aspectos que rodean a la música popular urbana. Además, (2) se observa el carácter multidimensional y holístico de los aprendizajes centrados en la creatividad y cooperativos. Los procesos de aprendizaje se han desarrollado a través de un ambiente de clase basado en la confianza mutua con un grado elevado de responsabilidad, determinado por el bienestar y la participación del alumnado. Además, dicho ambiente se ha caracterizado por un grado de motivación elevado y creciente de donde han emergido emociones positivas como una forma de acción social. En definitiva, el alumnado ha construido experiencias artísticas significativas a partir de sus vivencias en el aula. El contexto del aula ha dejado de ser el propio del aprendizaje habitual en Educación Secundaria y, de esta forma, esta experiencia dota de significatividad al aprendizaje. Por último, (3) hemos denotado una complejización de los procesos de aprendizaje derivado de la integración de los contenidos abordados, tal y como sugieren Cslovjecsek \& Zulauf (2018). A lo largo del proyecto, hemos comprobado, cómo el aprendizaje se ha producido gracias a un proceso holístico determinado por los siguientes factores: (a) la participación del alumnado, como desarrollo de principios democráticos y de justicia social en el aula; (b) la motivación desde el plano tanto individual como social expresado anteriormente; (c) las emociones positivas, desarrolladas de forma multidimensional en el aula de música; (d) el ambiente de clase y el bienestar del alumnado; (e) la identidad de los grupos desarrollada a través del proyecto didáctico.

Por todo esto, sugerimos una aproximación a la transdisciplinariedad artística en el ámbito de la Educación Secundaria, derivada de la propuesta de Hirsch Hadorn et al. (2008). En este sentido, la transdisciplinariedad quedaría definida por: (1) la integración y conexión epistemológica, con una cierta unificación metodológica desde la perspectiva de la enseñanza. Es decir, desde al menos dos asignaturas, con un tema unificado, y desde una serie de principios pedagógicos compartidos; (2) la participación activa del alumnado y una serie de participantes; (3) la conexión del aprendizaje con el mundo real; (4) diversos participantes implicados, que asumirían una variedad de roles.

A lo largo del proyecto hemos vivido un proceso enriquecedor y emocionante de transformación en el aula. Su ejemplo más concreto son las palabras del alumnado, nuestro mejor argumento: 
He aprendido a ser mejor compañera, y a escuchar a todos ya que todos aportan y hacen que sea mejor

He aprendidoque tod@s somos iguales

He aprendido a que si le dedicas tiempo y te esfuerzas por hacer lo que quieres, lo puedes conseguir

Este proyecto ha ayudado a que haya sido el mejor curso de mi vida

Este año música ha sido increíble, ya que tener un grupo de música ha sido un sueño para mí.

\section{REFERENCIAS BIBLIOGRÁFICAS}

Acaso, M. (2013). Reduvolution. Madrid: Paidós Ibérica.

Adams, R., Gibson, S. \& Müller Arisona, S. (Eds). (2008). Transdisciplinary digital art: Sound, vision and the new screen. Springer.

Bascia, N. \& Hargreaves, A. (Eds)(2013). The Sharp Edge of Educational Change: Teaching, Leading and the Realities of Reform. New York: Routledge.

Bunard, P. y Younker, B. A. (2008). Investigating children's musical interactions within the activities systems of group composing and arranging: An application of Engeström's Activity Theory. International Journal of Educational Research, 47(1), 60-74. https://doi.org/10.1016/j. ijer.2007.11.001

Burnard, P. \& Dragovic, T. (2014). Collaborative creativity in instrumental group learning as a site for enhancing pupil wellbeing. Cambridge Journal of Education: Special Issue 'Creativity and Wellbeing'. https://doi.org/10.1080/0305764X.2014.934204.

Bresler, L. (2006). Paradigmas cualitativos en la investigación en educación musical. En M. Díaz (ed.), Introducción a la investigación en educación musical. Madrid: Enclave Creativa.

Bunge, M. (1980). Epistemología. Curso de Actualización. Barcelona: Editorial Ariel.

Burnard, P. (2012). Musical Creativities in Practice. Oxford: Oxford University Press.Burnard, P., Craft, A., Cremin, T., Duffy, B., Hanson, R., Keene, J., Haynes, L. y Burns, D. (2006). Documenting "possibility thinking": A journey of collaborative enquiry. International Journal of Early Years Education, 14(3), 243-262. https://doi.org/10.1080/09669760600880001

Burnard, P., Apelgren, B. M. y Cabaroglu, N. (2015). Researching teachers: Teachers researching as teaching practice. En P. Burnard, B. M. Apelgren y N. Cabaroglu (Ed.), Transformative teacher research: Theory and practice for the C21st. Boston: Sense Publishers.

Burger, P. \& Kamber, R. (2003). Cognitive Integration in Transdisciplinary Science. Issues in Integrative Studies, (21), 43-73.

Cochran-Smith, M y Litle, S. L. (2009): Inquiry as Stance: Practitioner Research for the Next Generation. New York: Teachers College Press.

Comunidad de Madrid. 2018. Datos población. Madrid: Comunidad de Madrid.

Cross, I., Laurence, F. y Rabinowitch, T. (2012). Empathy and Creativity in Group Musical Practices: Towards a Concept of Emphatic Creativity. En G. Welch y G. McPherson (eds), The Oxford Handbook of music education (pp. 337-353). Nueva York: Oxford University Press.

Csikszentmihalyi, M. (1999). Implications of a systems perspective for the study of creativity. En R. J. Sternberg (Ed.), The handbook of creativity (pp. 313-335). New York: Cambridge University Press.

Cslovjecsek, M. \& Zulauf, M. (Eds.). (2018). Integrated Music Education. Bern, Switzerland: Peter Lang AG.

Dewey, J. (1934). Art as experience. Rahway, NJ: The Barnes Foundation Press. . (1995). Democracia y Educación. Madrid, Morata. 
Drake, S. M. \& Burns, R. C. (2004). Integrated Curriculum, Meeting Standards Through.Virginia USA: Association for Supervision and Curriculum Development.

Engeström, Y. (1987). Learning by expanding: An activity theoretical approach to developmental research. Helsinki: Orienta-Konsultit.

Engestrom, Y. (1999). Activity theory and individual and social transformation. En Y. Engestrom, R. Miettinen \& R. L. Punamaki (Eds.), Perspectives on activity theory (pp. 19-38). Cambridge: Cambridge University Press.

Feldman, D. H., Csikszentmihalyi, M. y Gardner, H. (1994). Changing the world: A framework for the study of creativity. Westport, CT: Praeger.

Fernández Enguita, M. (2016). La educación en la encrucijada. España: Fundación Santillana.

Finnish National Board of Education. (2016): National Core Curriculum for General upper secondary Schools.

Freire, P. (2003). Pedagogía del Oprimido (17 ed). Madrid: Siglo Veintiuno de españa editores.

García Díaz, D. (2017). Hacia una antropología integral. Un estudio a la luz de dos propuestas epistemológicas contemporáneas: Jacques Maritain y Basarab Nicolescu. Tesis Doctoral, Madrid: Universidad Francisco de Vitoria,

Glaveanu, V. (2018). Educating which creativity? Thinking Skills and Creativity, 27, 25-32. https:// doi.org/10.1016/j.tsc.2017.11.006

Greeno, J. \& Engeström, Y. (2014). Learning in Activity. In R. Sawyer (Ed.), The Cambridge Handbook of the Learning Sciences (Cambridge Handbooks in Psychology, pp. 128-148). Cambridge: Cambridge University Press. https://doi:10.1017/CBO9781139519526.009

Heron, J. (1996). Co-operative Inquiry: Research Into the Human Condition. London: Sage.

Hirsch Hadorn, G., Hoffmann-Riem, H., Biber-Klemm, S., Grossenbacher-Mansuy, W., Joye, D., Pohl, C., Wiesmann, U. \& Zemp, E. (Eds.) (2008). Handbook of Transdisciplinary Research, Dordrecht: Springer.

Jantsch, E. (1972). Towards Interdisciplinarity and Transdisciplinarity in Education and Innovation. In: L. Apostel et al. (eds.), Problems of Teaching and Research in Universities, (pp. 97-121). Organisation for Economic Cooperation and Development (OECD) and Center for Educational Research and Innovation (CERI): Paris.

Lage-Gómez, C. (2016). Música e imágenes un estudio de investigación acción sobre creación musical en tercero de Educación Secundaria obligatoria (Tesis Doctoral). Universidad Complutense de Madrid, Madrid.

Lage-Gómez, C. \& Cremades-Andreu, R. (2018). Pintando sonidos a través de la Improvisación Colectiva en Educación Secundaria. Revista Electrónica Complutense de Investigación En Educación Musical - RECIEM, 15(0), 61-82. https://doi.org/10.5209/reciem.55090 . (2019). Group improvisation as dialogue: Opening creative spaces in secondary music education. Thinking Skills and Creativity, 31. https://doi.org/10.1016/j.tsc.2018.12.007

. (2020). Theorising 'participatory creativity' in music education: unpacking the whole process at a Spanish secondary school. Music Education Research, 22(1), 54-67. https://doi.org /10.1080/14613808.2019.1703922

Lapidaki, E., Rokus de Groot, R. \& Stagkos, P. (2012). Comunal creativity as sociomusical practice. En G. Welch y G. McPherson (eds), The Oxford Handbook of music education (pp. 371-378), NY: Oxford University Press.

Marshall, J. (2014). Transdisciplinarity and Art Integration: Toward a New Understanding of ArtBased Learning across the Curriculum. Studies in Art Education, 55(2), pp. 104-127. https://doi. org/10.1080/00393541.2014.11518922

Miell, D. \& Littleton, K. (eds) (2004). Collaborative Creativity: Contemporary Perspectives. London: Free Association Books.

Marjanen, K. \& Cslovjecsek, M. (2013). Transversal processes - learning more about the teaching profession through music. The European Journal of Social \& Behavioural Sciences (EJSBS) VII Design in Mind, 1234-1243. 
Mittelstrass, J. (2011). On transdisciplinarity. Trames, 15(4), 329-338.

Morin, E. (1999). Los siete saberes necesarios para la educación del futuro (Trad. Mercedes Vallejos Gómez). París, Francia: Santillana/UNESCO.

Nicolescu, B. (2012). Transdisciplinarity: The hidden third, between the subject and the object. Human and Social Studies, 1(2), 13-28.

Perignat, E. \& Katz-Buonincontro, J. (2019). STEAM in practice and research: An integrative literature review. Thinking Skills and Creativity, 31, 31-43. https://doi.org/10.1016/j.tsc.2018.10.002

Klein, J. T., Grossenbacher-Mansuy, W., Häberli, R., Bill, A., Scholz, R.W. \& Welti, M. (2001). Transdisciplinarity: Joint Problem Solving among Science. An Effective Way for Managing Complexity. Basel: Birkhäuser Verlag.

Park, J. \& Son, J. (2010). Transitioning toward transdisciplinary learning in a multidisciplianaryenvironment. International Journal of Pedagogies and Learning, 6(1), 82-93.

Piaget, J. (1973). La situación de las ciencias del hombre dentro del sistema de las ciencias, en C. Castrillo (Ed.). Tendencias de la investigación en las ciencias sociales. (pp. 44-121). Madrid: Alianza Editorial.

Rice, P. \& Ezzy, D. (1999). Qualitative Research Methods: A Health Focus Melbourne: Oxford University Press.

Saether, E., Mbye, A. \& Shayesteh, R. (2012). Intercultural Tensions and Creativity in Music. En G. Welch y G. McPherson (Eds.), The Oxford Handbook of music education (pp.354-370). NY: Oxford University Press.

Sawyer, R. K. \& DeZutter, S. (2009). Distributed creativity: How collective creations emerge from collaboration. Psychology of Aesthetics, Creativity, and the Arts, 3(2), 81-92. https://doi.org/ 10.1037/a0013282

Seddon, F. A. (2012): Empathetic creativity in music making", en O. Odena (Ed), Musical creativity: insights from music education (pp. 133-147), Farnham: Ashgate.

Soares, J. (2012). The nature of the engagement of Brazilian adolescents in composing activities, en O. Odena (Ed), Musical Creativity: Insights from Music Education Research (pp. 113-132). Ashgate, Farnham, UK: Ashgate.

Sorieau, E. (1998). La correspondencia de las artes. México, D.F: Fondo de Cultura Económica.

Soublis Smyth, T. (2017). Transdisciplinary Pedagogy: A Competency Based Approach for Teachers and Students to Promote Global Sustainability. Journal of Interdisciplinary Studies in Education, $5(2), 64-72$

Viladot, L., Hilton, C., Casals, A., Saunders, J., Carrillo, C., Henley, J., Cristina González-Martín, C., Prat, M. \& Welch, G. (2018). The integration of music and mathematics education in Catalonia and England: perspectives on theory and practice. Music Education Research, 20(1), 7182. https://doi.org/10.1080/14613808.2017.1290595

Webster, P. (2002). Creative thinking in music: advancing a model. En T. Sullivan y L. Willingham (Eds.), Creativity and music education (pp.16-33). Edmonton: Canadian Music Educator's Association.

Wegerif, R. (2019). Dialogic education. In Oxford Research Encyclopedia of Education. Oxford University Press. doi: http://dx.doi.org/10.1093/acrefore/9780190264093.013.396

Withall, J. (1989). Enseñanza centrada en el profesor y enseñanza centrada en el alumno. En: T. Husén, T. N. Postlethwaite, (Dirs.). Enciclopedia Internacional de la Educación, 4, VicensVives/MEC, Barcelona/Madrid, 2153-2163. 